\title{
Observing Slow Crustal Movement in Residential User Traffic
}

\author{
Kenjiro Cho \\ IIJ
}

\author{
Kensuke Fukuda \\ NII / PRESTO JST
}

\author{
Hiroshi Esaki \\ Univ. of Tokyo
}

\author{
Akira Kato \\ Keio Univ.
}

\begin{abstract}
It is often argued that rapidly increasing video content along with the penetration of high-speed access is leading to explosive growth in the Internet traffic. Contrary to this popular claim, technically solid reports show only modest traffic growth worldwide. This paper sheds light on the causes of the apparently slow growth trends by analyzing commercial residential traffic in Japan where the fiber access rate is much higher than other countries. We first report that Japanese residential traffic also has modest growth rates using aggregated measurements from six ISPs. Then, we investigate residential per-customer traffic in one ISP by comparing traffic in 2005 and 2008, before and after the advent of YouTube and other similar services. Although at first glance a small segment of peer-to-peer users still dictate the overall volume, they are slightly decreasing in population and volume share. Meanwhile, the rest of the users are steadily moving towards rich media content with increased diversity. Surely, a huge amount of online data and abundant headroom in access capacity can conceivably lead to a massive traffic growth at some point in the future. The observed trends, however, suggest that video content is unlikely to disastrously overflow the Internet, at least not anytime soon.
\end{abstract}

\section{Categories and Subject Descriptors}

C.2.3 [Computer-Communication Networks]: Network Operations - Network monitoring

\section{General Terms}

Measurement, Management

\section{Keywords}

traffic growth, ISP traffic, residential broadband

Permission to make digital or hard copies of all or part of this work for personal or classroom use is granted without fee provided that copies are not made or distributed for profit or commercial advantage and that copies bear this notice and the full citation on the first page. To copy otherwise, to republish, to post on servers or to redistribute to lists, requires prior specific permission and/or a fee.

ACM CoNEXT 2008, December 10-12, 2008, Madrid, SPAIN

Copyright 2008 ACM 978-1-60558-210-8/08/0012 ...\$5.00.

\section{INTRODUCTION}

For the last few years, video content has become increasingly popular among Internet users. Today's Internet users casually view and share video content, benefited from the penetration of broadband access and easy-to-use video services on the Net. On the other hand, video and other rich media content are by orders of magnitude larger in amount than traditional web content. Thus, it is often projected that rapidly increasing video content is leading to explosive growth in the Internet traffic.

Despite of this popular claim, technical sources report only modest traffic growth worldwide. Odlyzko estimates the Internet traffic growth rate in 2007 to be about $50 \%$ to $60 \%$ in the U.S. and worldwide[19]. Cisco's recent report also estimates that worldwide Internet traffic growth has been around $50 \%$ per year over the last few years[6].

The growth of the Internet traffic volume is one of the key factors driving research, development and investment in data communication technologies and infrastructure. With the annual growth rate of $100 \%$, it grows 1000-fold in 10 years, while with 50\%, it grows only 58-fold. Hence, a difference in growth rate has a considerable impact in the long run. In order to accommodate innovations brought by empowered end users, what is crucial is not just growth rate but the balance among demands, technological advances and investment in infrastructure. If the growth is underestimated, we may not have capacity enough to handle new demands. If overestimated, investment in technologies and capacity may be only wasted.

This paper investigates the traffic growth in Japanese residential broadband traffic. Japan is one of the highest fiber access penetration countries in the world[20], and the number of Fiber-To-The-Home (FTTH) subscribers has exceeded that of DSL subscribers as shown in Figure 1[29]. Meanwhile, the total number of broadband subscribers has reached $56 \%$ of households and increased by only $5 \%$ in 2007[29]. However, even with the abundant access capacity, the traffic growth rate at Japanese major Internet eXchange points (IXes) has re- 


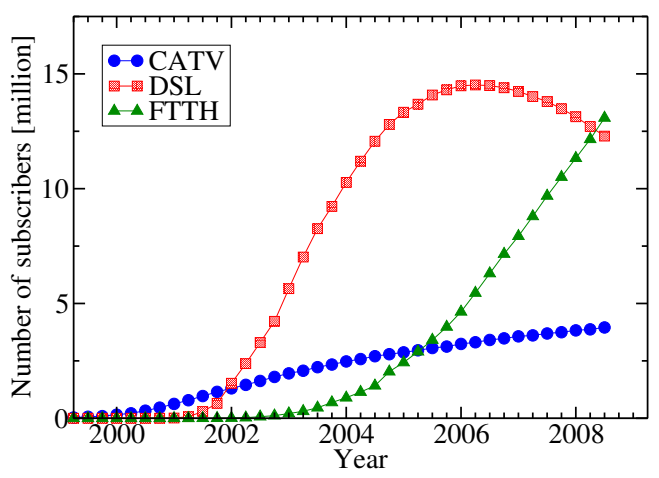

Figure 1: Number of residential broadband subscribers in Japan: 29.3 million total broadband subscribers, 13.1 million for FTTH, 12.3 million for DSL and 3.9 million for CATV as of June 2008.

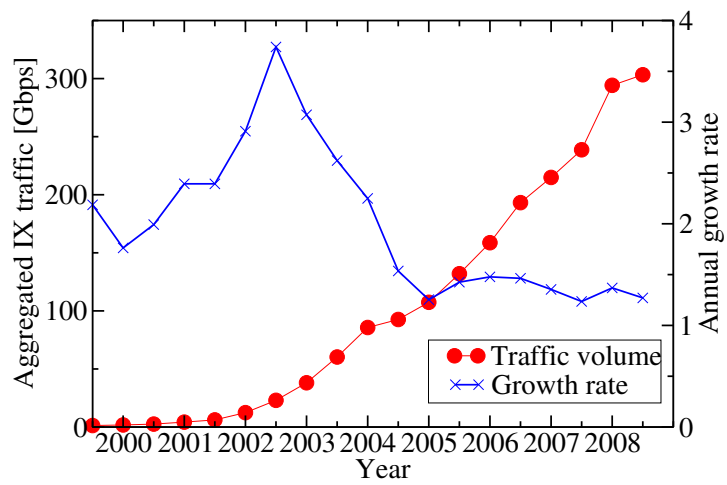

Figure 2: The aggregated peak rate and its growth rate of the major Japanese IXes

mained under $50 \%$ for the last few years as depicted in Figure 2. The plot shows aggregated peak traffic and its growth rate at major IXes, namely JPIX[15], JPNAP[16] and NSPIXP[18], and illustrates multiplicative growth of traffic where the volume is doubling roughly every two years since 2005. However, the growth rate does not seem to be affected significantly by the advent of popular video services in 2006.

We have been studying commercial backbone traffic in Japan with support from six ISPs covering $42 \%$ of the Japanese backbone traffic. (We started with seven ISPs but two were merged in 2006.) In our previous work[3], we reported that the backbone in 2005 was dominated by residential user-to-user traffic.

In this paper, we try to answer a key question: what is the macro level impact of video and other rich media content on the traffic growth at the moment? We shed light on the causes of the apparently slow growth trend by detailed analyses of commercial residential traffic.

We followed the methodology used in our previous work[3], and used two different data sets. The first set was collected by aggregating interface counters of edge routers from the six ISPs. The other set was collected by Sampled NetFlow[4] from one of the ISPs for detailed per-customer analysis.

We first report that Japanese residential broadband (RBB) traffic also has a modest average annual growth rate of $27 \%$ over the last three years by means of aggregated measurements from the six ISPs. Then, we further investigate residential per-customer traffic in one of the ISPs by comparing traffic in 2005 and 2008 that is before and after the advent of YouTube[31] and other similar video services. Although at first glance a small segment of peer-to-peer users still dictate the overall traffic volume, it is decreasing in both population share and volume share. At the same time, the rest of the users are steadily moving towards rich media content with increased diversity.

The current traffic is heavily affected by an eruption of peer-to-peer applications but the crust underneath is also slowly rising with video and other rich media content. The crustal movement is slow at the macro level so that it is unlikely to cause a major quake in the near future.

\section{RELATED WORK}

This is a sequel of our previous work[3] in which we compared traffic usage between fiber and DSL users, and between heavy-hitters and the rest of the users. The focus was on heavy-hitters in residential traffic but this paper focuses on ordinary residential users. One of the contributions of this paper is to illustrate the impacts of peer-to-peer and rich media traffic on the current traffic growth. Another contribution is to quantify changes in traffic usage of ordinary residential users.

The traffic growth rate is reported to be slowing down in recent years. Odlyzko monitors over 100 publicly available traffic statistics, and estimates the Internet traffic growth rate[19]: the U.S. Internet traffic grew at around $100 \%$ in early 1990 , jumped up to $1,000 \%$ in 1995-1996 driven by world wide web and user expansion, went back to $70-150 \%$ in $1997-2003$, and settled down to around 50\% in 2004-2007. Cisco's reports[6, $5]$ are based on traffic data from its provider customers combined with analyst projections, and also estimate that worldwide Internet traffic growth has been around $50 \%$ per year over the last few years. the Japanese IX traffic data in Figure 2 also shows that the growth rate has been stable at around $30 \%$ over the last four years. These estimates do not have details on user distributions so that our detailed analyses are complementary to these reports.

There is little solid work in literature that tries to measure commercial residential traffic. Studies on ADSL networks $[23,27]$ are similar to our per-customer analy- 
sis in monitoring access lines and comparing traffic volumes among data sets but their focus is on file-sharing traffic. It is reported that the average daily traffic volume of heavy-hitters is $470 \mathrm{MB}$ for inbound and 760MB for outbound, and that of non heavy-hitters is $9 \mathrm{MB}$ for inbound and $27 \mathrm{MB}$ for outbound in 2006[27].

There are numerous studies on traditional web traffic, peer-to-peer traffic and video traffic. Many of them try to characterize traffic at the flow level. Peer-to-peer traffic is highly variable and skewed among participating nodes $[24,26,12]$, and exhibit behavior considerably different from traditional web traffic[11, 1]. A recent study on YouTube traffic[10] by monitoring an academic network compares characteristics of video content with traditional web content. The mean and median size of video content are $10 \mathrm{MB}$ and $8 \mathrm{MB}$, and the mean and median transfer rates are $394 \mathrm{kbps}$ and $328 \mathrm{kbps}$. Another study[17] characterizes streaming media files stored online by crawling web sites, and compares different types of video content. Many studies report that flow size and duration follow heavy-tailed distributions $[25,32,2]$, and discuss elephants and mice in flows. However, there exists few previous work focusing on the evolution of traffic volume per user, especially for residential users.

Regarding a shift in traffic mix, Cisco's report[6] has statistics about a traffic mix shift in consumer traffic; peer-to-peer file-sharing traffic grew $29 \%$ in volume but the volume share dropped from $60 \%$ in 2006 to $51 \%$ in 2007. The volume share of video content not including peer-to-peer file-sharing traffic grew from $12 \%$ in 2006 to $22 \%$ in 2007 , and is projected to be $50 \%$ in 2012 . The estimated peer-to-peer traffic share is smaller than our results and other reports (e.g., 86-93\% in an academic backbone[14]). Cisco's projection is calculated by projected online hours per user multiplied by the average MB per hour for different traffic types. Our findings in traffic distributions could provide new insights into these traditional projection methods.

\section{AGGREGATED TRAFFIC ANALYSIS}

This section examines the traffic growth trends in Japan by means of aggregated measurements collected from six ISPs covering $42 \%$ of the Japanese backbone traffic. The results show that Japanese commercial traffic has modest growth rates over the last three years. In particular, the average annual growth rate of residential traffic has been 27\% from May 2005 through May 2008 .

\subsection{Measurement Methodology}

The ISPs we collaborate with collect interface counter values of almost all routers in their service networks via SNMP, and archive per-interface traffic logs using MRTG [22] or RRDtool [21]. Thus, it is possible for the ISPs to provide aggregated traffic information if they

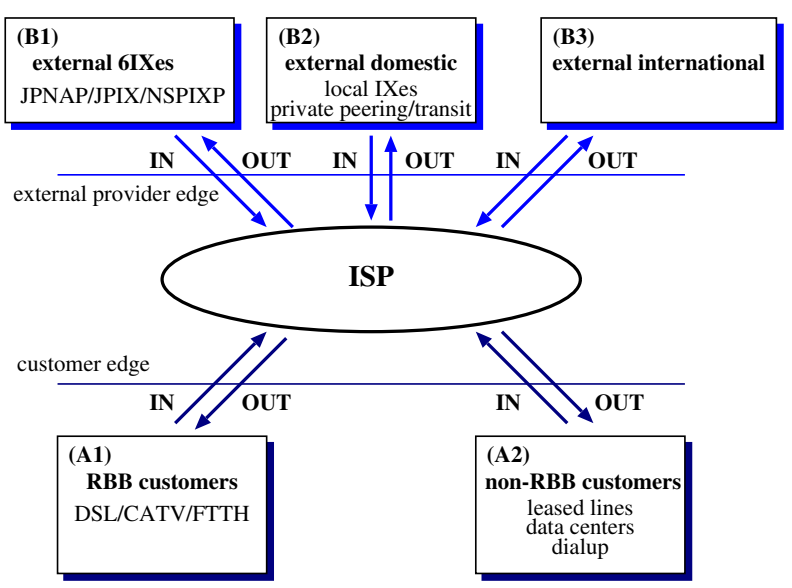

Figure 3: Five traffic groups for data collection at customer and external boundaries of an ISP

can classify router interfaces into a common set.

Our focus is on traffic crossing ISP boundaries which can be roughly divided into customer traffic, and external provider traffic such as peering and transit. For practical purposes, we selected the five traffic groups in Figure 3 for data collection: (A1) RBB customers represent residential broadband customer lines. This group also includes small business customers using residential broadband access. (A2) non-RBB customers represent customer lines other than RBB customers, including leased lines, data centers, and dialup lines. This group includes RBB customers behind leased lines, e.g., second or third level ISPs, since ISPs do not distinguish them from other leased lines. (B1) external 6IXes represent links for 6 major IXes, namely JPIX, JPNAP and NSPIXP in both Tokyo and Osaka in order to compare measurements at these IXes as well as to know the traffic share of our measurement. (B2) external domestic represents domestic external provider links other than the 6IXes, including regional IXes, private peering and transit. We used the term "domestic" to indicate that both ends of a link are located in Japan. This group also includes domestic peering with global ASes. (B3) external international represents international external provider links with one end point outside of Japan.

These groups are chosen by the existing operational practices of the participating ISPs because it is impossible to draw a strict line for grouping, e.g., residential/business and domestic/international, on the global Internet. We re-aggregate each ISP's aggregated logs, and only the resulting aggregated traffic is used in our study so as to not reveal the share of each ISP. Although the peak rate is often used for operational purposes, only the mean rate is collected since the peak rate is not summable. 
In general, it is meaningless to simply sum up traffic values from multiple ISPs since a packet could cross ISP boundaries multiple times. Customer traffic is, however, summable because a packet crosses customer edges only once in each direction, when entering the source ISP and exiting the destination ISP. The numbers for external provider traffic are overestimated since a packet could be counted multiple times if it travels across intermediate ISPs. Nevertheless, the error should be negligible in this particular result since most of the ISPs in our data sets are peering, and thus, not providing transit to each other. Although one ISP is identified to have double counts for the transit link, it is compensated in the results by data provided separately for the double count adjustment.

Each ISP provided month-long traffic logs aggregated for each traffic group by a log aggregation tool we developed. This allows ISPs not to disclose the internal structure of their network or unneeded details of their traffic. The final results were obtained by aggregating all traffic logs provided by the six ISPs.

The time resolution of the logs is 2 hours since it was the highest common factor for month-long data in MRTG and RRDtool; 2-hour boundaries in UTC fall on odd hours in Japanese Standard Time $(\mathrm{UTC}+9)$. Note that, throughout the paper, inbound and outbound are presented from the ISPs' point of view as depicted in Figure 3.

The data for each month was separately collected, and consistency such as each ISP's share, differences from the previous measurements, and the coverage of the IX traffic was examined. Then, the aggregated results were provided to the ISPs so that each ISP can compare and check its own data against the aggregated results. In addition, a face-to-face meeting with representatives from the participating ISPs is held after each data collection to check and discuss the results.

Monthly traffic logs with two-hour resolution allow us to identify major changes in each ISP's traffic. When such changes are found, we contact the ISP to confirm the cause of the change, e.g., a network reconfiguration, an outage, missing SNMP data, or a mis-classification of interface counter logs. If necessary, we ask the ISP to correct and update the data.

\subsection{Data Sets}

We analyzed month-long traffic logs from six major Japanese ISPs for the last five years; September, October, November in 2004, May and November from 2005 through 2008. After the initial trials over three months, we decided to collect data only twice a year to reduce the workload of the participating ISPs.

\subsection{Growth of Traffic}

The monthly average rates in bits/second of the traf-

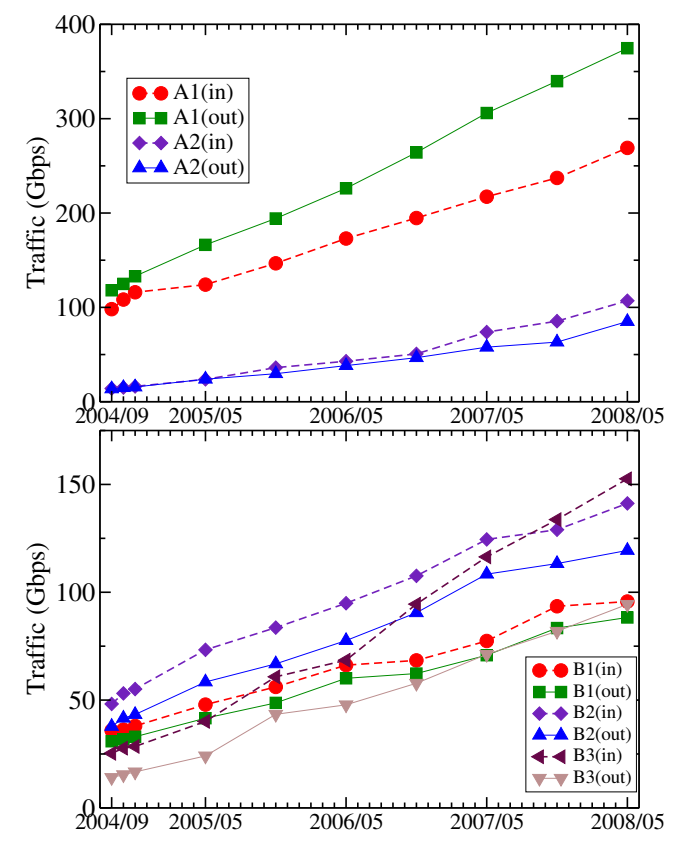

Figure 4: Traffic growth: customer traffic (top) and external provider traffic (bottom)

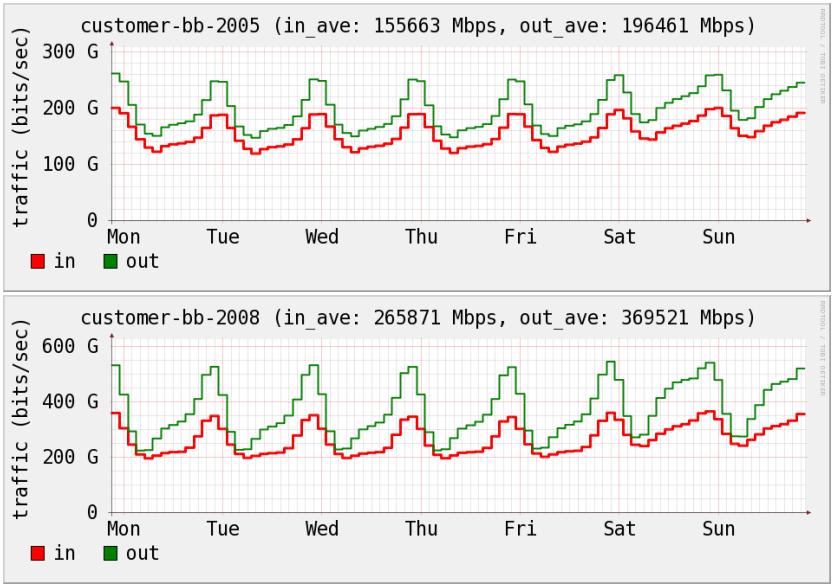

Figure 5: Residential broadband customer traffic in May 2005 (top) and May 2008 (bottom)

fic groups are shown in Tables 1 and their growth is illustrated in Figure 4. Between May 2005 and May 2008 , the average annual growth rate of (A1) RBB customers was $26 \%$ for inbound, $28 \%$ for outbound, and $27 \%$ for the combined volume. The difference between inbound and outbound has widened over the years.

Figure 5 compares weekly RBB customer traffic (A1) in May 2005 and in May 2008. For weekly data analysis, we took the averages of the same weekdays in a month but excluded holidays since holiday traffic patterns are closer to weekends'. The diurnal pattern re- 
Table 1: Monthly average rates of aggregated customer and external traffic (bps)

\begin{tabular}{|c|c|c|c|c|c|c|c|c|c|c|c|}
\hline & & \multirow{2}{*}{\multicolumn{2}{|c|}{$\begin{array}{l}\text { (A1)customer-RBB } \\
\text { (6 ISPs) } \\
\text { inbound outbound }\end{array}$}} & \multicolumn{2}{|c|}{$\begin{array}{l}\text { (A2)customer-non-RBB } \\
\text { (4 ISPs) }\end{array}$} & \multicolumn{2}{|c|}{$\begin{array}{l}\text { (B1)ext-6ix } \\
\text { (6 ISPs) }\end{array}$} & \multicolumn{2}{|c|}{$\begin{array}{l}\text { (B2)ext-domestic } \\
\text { (6 ISPs) }\end{array}$} & \multicolumn{2}{|c|}{$\begin{array}{c}\text { (B3)ext-international } \\
\text { (6 ISPs) }\end{array}$} \\
\hline & & & & inbound & outbound & inbound & outbound & inbound & outbound & inbound & outbound \\
\hline \multirow[t]{3}{*}{2004} & Sep & $98.1 \mathrm{G}$ & $111.8 \mathrm{G}$ & $14.0 \mathrm{G}$ & $13.6 \mathrm{G}$ & $35.9 \mathrm{G}$ & $30.9 \mathrm{G}$ & $48.2 \mathrm{G}$ & $37.8 \mathrm{G}$ & $25.3 \mathrm{G}$ & $14.1 \mathrm{G}$ \\
\hline & Oct & $108.3 \mathrm{G}$ & $124.9 \mathrm{G}$ & $15.0 \mathrm{G}$ & $14.9 \mathrm{G}$ & $36.3 \mathrm{G}$ & $31.8 \mathrm{G}$ & $53.1 \mathrm{G}$ & $41.6 \mathrm{G}$ & $27.7 \mathrm{G}$ & $15.4 \mathrm{G}$ \\
\hline & Nov & $116.0 \mathrm{G}$ & $133.0 \mathrm{G}$ & $16.2 \mathrm{G}$ & $15.6 \mathrm{G}$ & $38.0 \mathrm{G}$ & $33.0 \mathrm{G}$ & $55.1 \mathrm{G}$ & $43.3 \mathrm{G}$ & $28.5 \mathrm{G}$ & $16.7 \mathrm{G}$ \\
\hline \multirow[t]{2}{*}{2005} & May & $134.5 \mathrm{G}$ & $178.3 \mathrm{G}$ & $23.7 \mathrm{G}$ & $23.9 \mathrm{G}$ & $47.9 \mathrm{G}$ & $41.6 \mathrm{G}$ & $73.3 \mathrm{G}$ & $58.4 \mathrm{G}$ & $40.1 \mathrm{G}$ & $24.1 \mathrm{G}$ \\
\hline & Nov & $146.7 \mathrm{G}$ & $194.2 \mathrm{G}$ & $36.1 \mathrm{G}$ & $29.7 \mathrm{G}$ & $54.0 \mathrm{G}$ & $48.1 \mathrm{G}$ & $80.9 \mathrm{G}$ & $68.1 \mathrm{G}$ & $57.1 \mathrm{G}$ & $39.8 \mathrm{G}$ \\
\hline \multirow[t]{2}{*}{2006} & May & $173.0 \mathrm{G}$ & $226.2 \mathrm{G}$ & $42.9 \mathrm{G}$ & $38.3 \mathrm{G}$ & $66.2 \mathrm{G}$ & $60.1 \mathrm{G}$ & $94.9 \mathrm{G}$ & $77.6 \mathrm{G}$ & $68.5 \mathrm{G}$ & $47.8 \mathrm{G}$ \\
\hline & Nov & $194.5 \mathrm{G}$ & $264.2 \mathrm{G}$ & $50.7 \mathrm{G}$ & $46.7 \mathrm{G}$ & $68.4 \mathrm{G}$ & $62.3 \mathrm{G}$ & $107.6 \mathrm{G}$ & $90.5 \mathrm{G}$ & $94.5 \mathrm{G}$ & $57.8 \mathrm{G}$ \\
\hline \multirow[t]{2}{*}{2007} & May & $217.3 \mathrm{G}$ & $306.0 \mathrm{G}$ & $73.8 \mathrm{G}$ & $57.8 \mathrm{G}$ & $77.4 \mathrm{G}$ & $70.8 \mathrm{G}$ & $124.5 \mathrm{G}$ & $108.4 \mathrm{G}$ & $116.4 \mathrm{G}$ & $71.2 \mathrm{G}$ \\
\hline & Nov & $237.2 \mathrm{G}$ & $339.8 \mathrm{G}$ & $85.4 \mathrm{G}$ & $63.2 \mathrm{G}$ & $93.5 \mathrm{G}$ & $83.4 \mathrm{G}$ & $129.0 \mathrm{G}$ & $113.3 \mathrm{G}$ & $133.7 \mathrm{G}$ & $81.8 \mathrm{G}$ \\
\hline 2008 & May & $269.0 \mathrm{G}$ & $374.7 \mathrm{G}$ & $107.0 \mathrm{G}$ & $85.0 \mathrm{G}$ & $95.7 \mathrm{G}$ & $88.3 \mathrm{G}$ & $141.2 \mathrm{G}$ & $119.4 \mathrm{G}$ & $152.6 \mathrm{G}$ & $94.4 \mathrm{G}$ \\
\hline
\end{tabular}

flects home user activities, i.e., the traffic increases in the evening, and the peak hours are from 21:00 to 23:00. Weekends have larger daytime traffic although the peak rates are similar to weekdays. The outbound traffic to customers was only slightly larger than the inbound in 2005 but it becomes much larger in 2008 with larger fluctuations and with clearer peaks. The ratio of the bottom (the constantly flowing part) against the peak decreased from 0.63 to 0.56 for inbound, and from 0.59 to 0.43 for outbound. We found $63 \%$ of the residential traffic in 2005 was user-to-user [3], and believe that peerto-peer applications contribute to significant part of the upstream traffic as well as the constantly flowing part of the traffic in both directions. On the other hand, the larger increase in the outbound volume in 2008 seems to come from client-server type applications.

The ratio of the RBB customer volume against the total customer volume also decreased. The data for (A2) non-RBB customer traffic was obtained only from four ISPs so that it is not possible to directly compare (A1) with (A2). It is difficult for the other ISPs to distinguish external links from other links for historical reasons. Hence, we estimated the ratio of (A1) to (A) using only data from the four ISPs with both (A1) and (A2). The estimated ratio (A1)/(A1+A2) decreased from $59 \%$ in 2005 to $43 \%$ in 2008 for inbound and from $64 \%$ to $56 \%$ for outbound, which also indicates a decrease in user-to-user traffic share.

The total volume of (B2) external domestic traffic, mainly private peering, exceeds the volume for (B1) 6IXes and the difference is widening, probably because major ISPs are shifting from public peering to private peering to handle increasing demands. This result underlines a possible deviation in estimating nation-wide traffic only from IX traffic. However, the ratio of private peering could be overestimated in our results as private peering is usually exercised only between large ISPs. Another noticeable change is the increase in international traffic, especially for inbound. This is probably due to popular video services which do not have servers in Japan (e.g., YouTube).

Our IX traffic data (B1) has consistently represented about $42 \%$ of the inbound traffic measured and pub-
Table 2: Share of (B1) outbound to the IXes against the inbound measured by the IX (\%)

\begin{tabular}{ccc|cc|cc|cc|c}
\hline \multicolumn{3}{c|}{2004} & \multicolumn{2}{|c|}{2005} & \multicolumn{2}{c|}{2006} & \multicolumn{2}{c}{2007} & 2008 \\
Sep & Oct & Nov & May & Nov & May & Nov & May & Nov & May \\
\hline 41.5 & 41.9 & 41.6 & 42.0 & 41.4 & 43.1 & 41.5 & 42.4 & 41.8 & 42.6 \\
\hline
\end{tabular}

lished by the IXes as in Table 2 so that our data is considered to cover $42 \%$ of Japanese backbone traffic. If we assume this ratio to be the traffic share of the six ISPs, the total amount of residential traffic in Japan in May 2008 can roughly be estimated to be $632 \mathrm{Gbps}$ for inbound and $880 \mathrm{Gbps}$ for outbound, or $205 \mathrm{~PB} /$ month for inbound and $285 \mathrm{~PB} /$ month for outbound. These numbers are in line with Cisco's estimates of $226 \mathrm{~PB} /$ month for consumer traffic in Japan in 2008[6].

In summary, by comparing traffic in 2008 with 2005, we observed the larger download volume as well as larger evening volume in the residential traffic, the decreased share of residential traffic in the customer traffic, and the increased inbound volume in the international traffic. All of these, along with the volume comparable in size to peer-to-peer traffic, indicate an increase in video service content but the overall growth has been modest. A plausible explanation is that residential traffic is shifting from peer-to-peer file-sharing to video services. We will examine this hypothesis in the next section using per-customer measurements from one of the ISPs.

\section{PER-CUSTOMER TRAFFIC ANALYSIS}

To further analyze the behavior of residential traffic, sets of Sampled NetFlow data from 2004 to 2008 were obtained from one of the participating ISPs. Here, only residential broadband customer traffic is analyzed. In the data, one end of a flow is always the residential customer of the ISP but the other end is generally a customer of another ISP. This ISP has residential broadband customers over DSL and fiber but not over CATV.

By comparing the aggregated residential traffic graphs (e.g., Figure 5) with the ISP's corresponding graphs, we confirmed that the traffic characteristics are consistent. Therefore, we consider this ISP to be representative of the participating ISPs. 


\subsection{Measurement Methodology}

Data was collected from all edge routers accommodating residential broadband customers. Traffic volume is derived by dividing the measured volume by the sampling rate. In 2005, the sampling rate was $1 / 2048$ unanimously but, in $2008,45 \%$ of the routers, notably busy ones, have $1 / 8192,45 \%$ have $1 / 2048$, and $10 \%$ have $1 / 1024$, so as to keep up with the increased load of the routers. We believe it is still enough for analyzing user behaviors but there is a certain amount of sampling errors, especially for lightweight users.

\subsection{Data Sets}

Two types of week-long data sets are used for analysis. One type is daily inbound and outbound traffic volume of each customer obtained by matching customer IDs with the assigned IP addresses in NetFlow data. The data is also classified by customer's line type (DSL or fiber), and used to analyze per-customer behavior in Section 4.2 through 4.4 . The data was collected during April 12-18 in 2004, February 21-27 in 2005, July 9-15 in 2007, and June 2-8 in 2008. The other type is raw NetFlow data, and used to analyze protocol usage in Section 4.5. The data was collected during July 4-10 in 2005 and June 2-8 in 2008.

The main data sets for analysis are from 2005 and 2008 to observe the impact of video services. By comparing the 2008 data with the 2005 data, the total number of active users (unique customer IDs observed in the data set) increased by $94.7 \%$, and the total traffic volume increased by $187.0 \%$. The growth rate of the total traffic volume is larger than the aggregated result, because this ISP has expanded business in the residential broadband services.

The daily traffic volume per active user increased by $12.3 \%$ for inbound from $430 \mathrm{MB}$ in 2005 to $483 \mathrm{MB}$ in 2008 , and by $78.3 \%$ for outbound from $447 \mathrm{MB}$ to 797MB. The outbound volume became much larger in 2008. A large majority of the active users became fiber users in 2008 as shown in Table 3. The fiber user ratio increased from $46 \%$ in 2005 to $79 \%$ in 2008 . Accordingly, the total volume share of the fiber users increased from $79 \%$ in 2005 to $87 \%$ in 2008 .

Table 3: Ratio of fiber and DSL active users and their traffic volume share

\begin{tabular}{cc|c|c}
\hline & & active users (\%) & total volume (\%) \\
\hline 2005 & fiber & 46 & 79 \\
& DSL & 54 & 21 \\
\hline 2008 & fiber & 79 & 87 \\
& DSL & 21 & 13 \\
\hline
\end{tabular}

\subsection{Distribution of per-customer traffic}

The probability density functions (PDF) of daily traffic volume per customer in log-linear scale are shown in
Figure 6. The daily traffic volume is the average for the week, and the distribution is computed independently for inbound and outbound. The spikes at the left edge in the 2008 plots are due to the increased sampling rates.

When we look at the fiber users in 2005 (top middle), each distribution for inbound and outbound appears to consist of two roughly lognormal distributions where the logarithm of the variable is normally distributed. It is less clear in the other plots but the hypothesis of having two distinct distributions still holds in all the plots.

The distribution on the left includes the majority of users, and the outbound volume (download for users) is about ten times larger than the inbound volume. The other distribution on the right is high-volume users with symmetric inbound and outbound volumes. The two distinct distributions suggest that they have different mechanisms; most likely, the symmetric high-volume distribution is driven by the symmetric and aggressive nature of peer-to-peer file-sharing applications, and the asymmetric distribution for the majority of the users is driven by client-type applications (e.g., web browsers and video streaming viewers). For convenience, we call the symmetric high-volume group peer-type, and the other asymmetric group client-type. The vertical lines in the plots are at $100 \mathrm{MB} /$ day and $2.5 \mathrm{~GB} /$ day; the former is roughly at the center of the two groups for inbound, and the latter was used in our previous work as an inbound volume threshold for heavy-hitters.

The distributions of the client-type group have moved towards higher volume, and the outbound has grown much faster than the inbound. The mode for the total inbound shifted from $3.5 \mathrm{MB} /$ day in 2005 to $5 \mathrm{MB} /$ day in 2008, and the mode for the total outbound shifted from $32 \mathrm{MB} /$ day to $94 \mathrm{MB} /$ day. The distribution of the outbound volume becomes wider, suggesting increased diversity and larger influence by the tail.

On the other hand, the distributions of the peer-type group did not grow much. The mode of the distribution stays at around $2 \mathrm{~GB} /$ day, and the covered area (population share) has slightly decreased. As a result, the outbound peer-type distributions become harder to distinguish in the 2008 plots.

The modes of the distributions are similar in both fiber and DSL plots, indicating that the most frequent usage volume is similar but the peer-type group has a larger share in the fiber users.

The corresponding (complementary) cumulative distributions of daily traffic per user in 2005 and 2008 are shown on a log-log scale in Figure 7. The distributions are heavy tailed but there is a knee in the slope, at the top $4 \%$ of heavy-hitters using more than $2.5 \mathrm{~GB} /$ day (or 230kbits/sec) for the total users in 2005 as shown by the lines in the plots. The distribution for inbound did not change much in 2008 but that for outbound has 

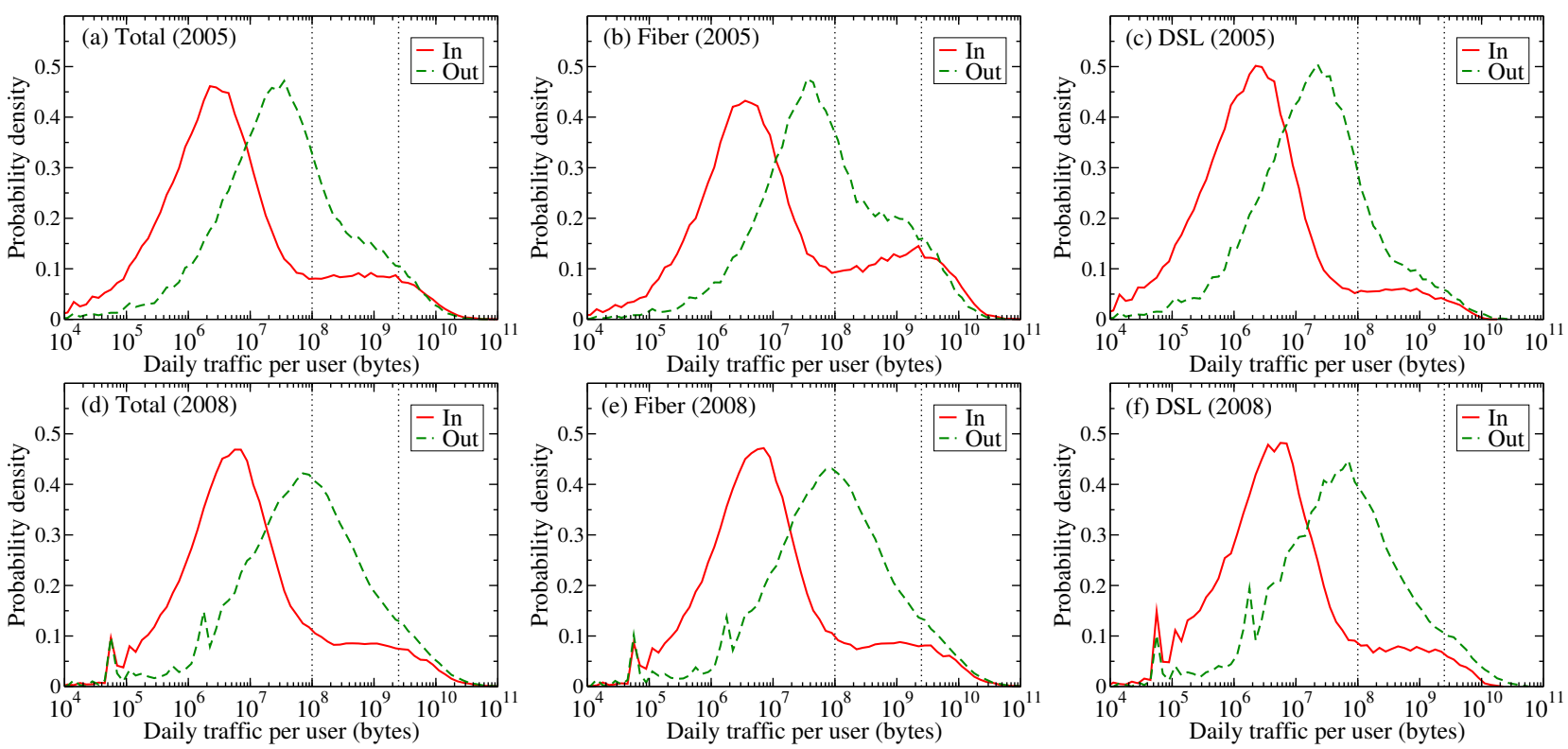

Figure 6: Probability density function of daily traffic per user: total (left), fiber users (middle), and DSL users (right) in 2005 (top) and 2008 (bottom).
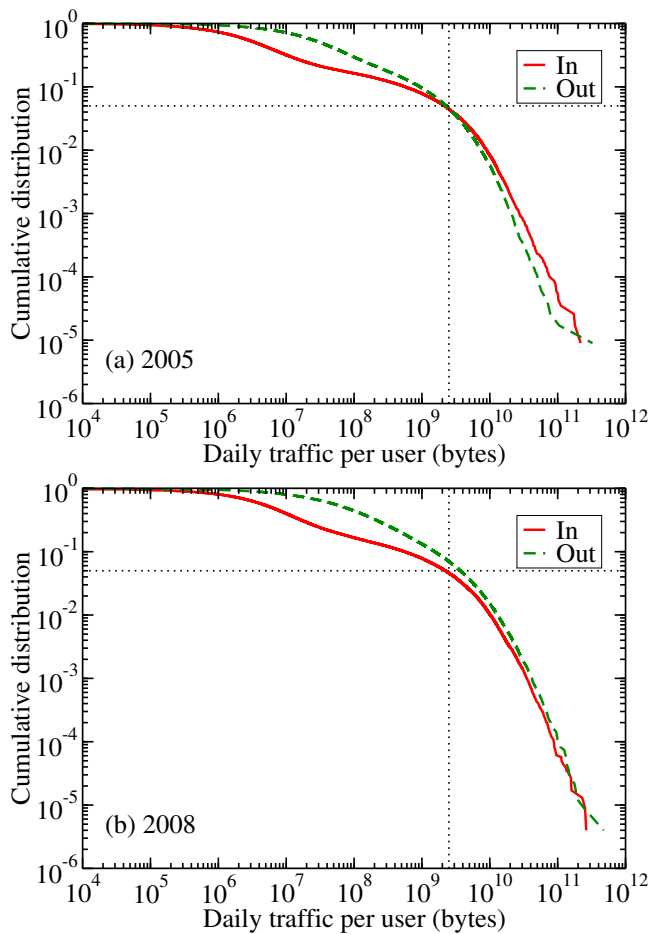

Figure 7: Cumulative distribution of daily traffic per user: 2005 (top) and 2008 (bottom).

moved to the right, suggesting visible growth only in the download volume. The inbound and outbound volumes of the tail become symmetric, probably because

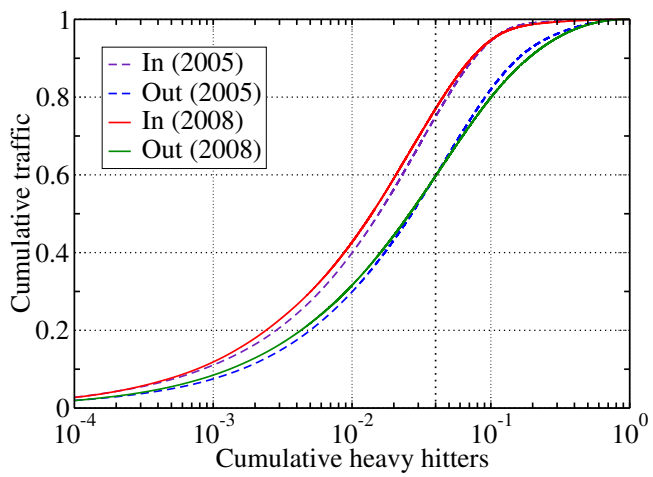

Figure 8: Cumulative distribution of traffic volume of top-ranking heavy-hitters: 2005 and 2008

the majority of the heavy-hitters become fiber users so that they do not need to compensate for the shortage of upstream bandwidth of DSL heavy-hitters.

Figure 8 shows the cumulative distribution of traffic volume consumed by top ranking heavy-hitters, computed independently for inbound and outbound. The plot shows a skewed traffic distribution among users; the top $\mathrm{N} \%$ of heavy-hitters use X\% of the total traffic. For example, the top $4 \%$ use $75 \%$ of the total inbound traffic, and $60 \%$ of the outbound. The distribution has not changed much from 2005 to 2008; the overall traffic is still dictated by a small group even with the increased traffic by the client-type users as the client-type users similarly have a long-tailed distribution. 

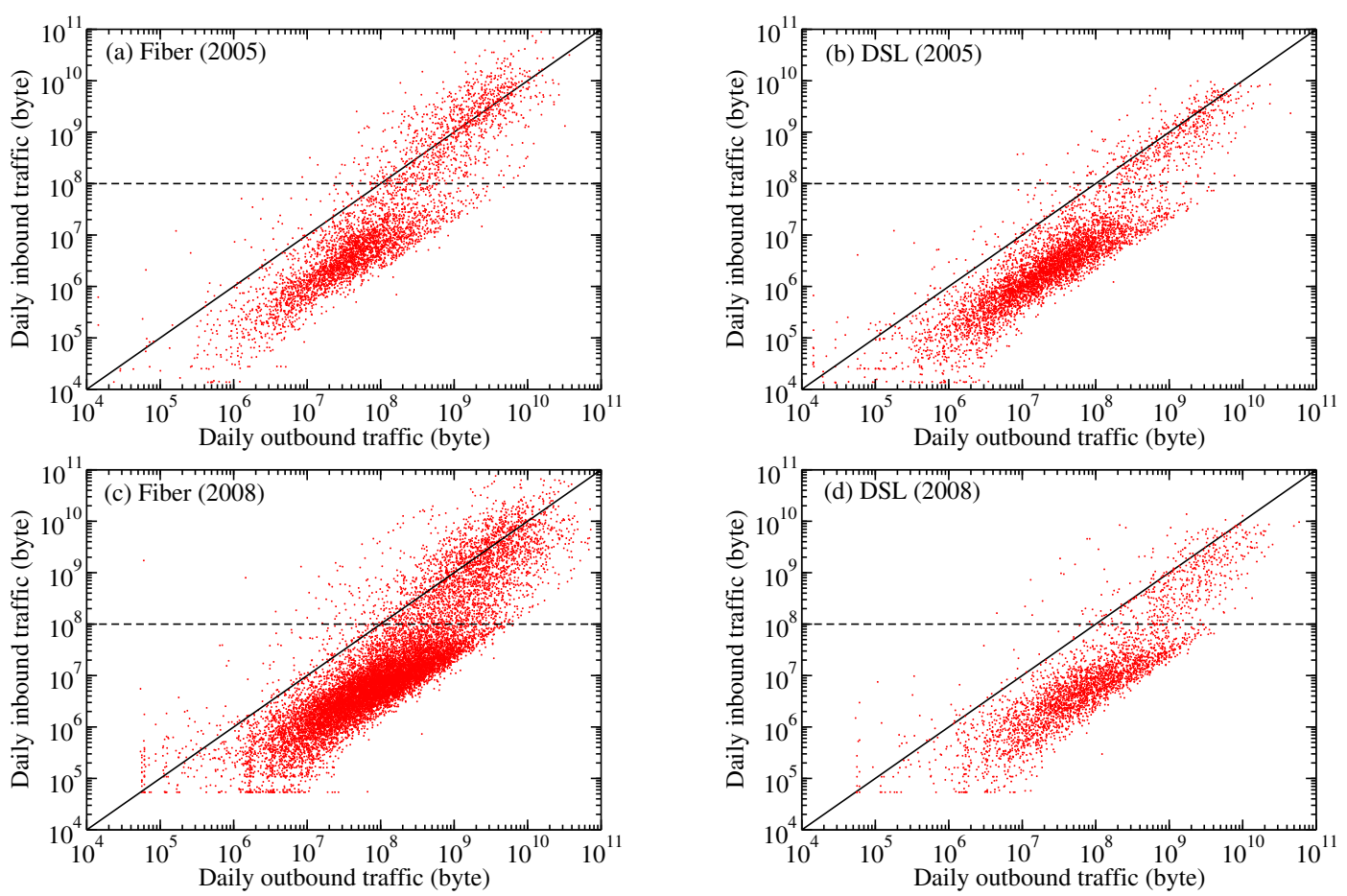

Figure 9: Correlation of inbound and outbound traffic volumes per user in one metropolitan prefecture: fiber (left) and DSL (right) in 2005 (top) and 2008 (bottom)

\subsection{Inbound and Outbound Correlation}

To observe the ratio of inbound and outbound volumes of each user, daily inbound and outbound volumes per user are shown in log-log scatter plots in Figure 9. These are taken from a metropolitan prefecture but the characteristics are common to all the prefectures. The number of plotted users is about 4300 for fiber and about 5400 for DSL in 2005 and about 14700 for fiber and about 3400 for DSL in 2008; a clear shift from DSL to fiber in 2008. The horizontal line is at $100 \mathrm{MB} /$ day for inbound that will be used to distinguish client-type users in Section 4.5.

In all the plots, the highest density cluster is below and parallel to the unity line where outbound volume (download for users) is about ten times larger than that of inbound, which corresponds to the client-type users. There exists another cluster in a higher volume region around the unity line, which corresponds to the peertype users.

The main difference between the fiber and DSL plots is heavy-hitters' population share; there are more heavyhitters in the fiber users as expected. It is also observed that there are heavy-hitters in the client-type group whose outbound volumes are comparable to that of heavy-hitters in the peer-type group, especially in the fiber users in 2008.

However, the boundary between client-type users and peer-type users as well as the boundary between heavyhitters and the rest of the users are not very clear. It is also observed that, across the entire traffic volume range, the inbound/outbound ratio varies greatly, up to 4 orders of magnitude in all the plots. It suggests that there exist diverse users with a different traffic mix of client-type and peer-type applications. That is, although it is possible to classify users into client-type and peer-type at the macro level, actual individual users have mixed traffic of both types.

\subsection{Protocol and Port Usage}

To observe differences in protocol and port usage between peer-type and client-type users, we classify the users simply by average daily inbound volume (upload for users) with the threshold of $100 \mathrm{MB} /$ day. This threshold is roughly at the center of the two distributions in the inbound PDFs in Figure 6, and classifies the users by the horizontal line in Figure 9. The population share of client-type users is $83.7 \%$ in 2005 and $83.3 \%$ in 2008 . In our previous work, we used $2.5 \mathrm{~GB} /$ day to distinguish heavy-hitters to focus on heavy-hitters but 100MB/day is used here to focus on client-type users.

To rank port numbers in TCP and UDP, we take the smaller of the source and destination ports of a flow. TCP ports are further divided into well-known ports that are smaller than 1024, and dynamic ports that are equal to or larger than 1024. Our purpose is to illustrate 
differences in port usage between the client-type and peer-type groups so that we simply use dynamic port traffic as a rough estimate of peer-to-peer traffic.

Table 4 shows the ranking of protocols and ports for the total users, the client-type users, and the peer-type users in 2005 and 2008. The ranking is similar for all the groups with minor differences in dynamic ports so that the table is ordered by the ranking of the total users in 2008.

Table 4: Protocol breakdown in percentage against the total volume: 2005 and 2008

\begin{tabular}{|c|c|c|c|c|c|c|}
\hline \multirow[b]{2}{*}{ protocol port } & \multicolumn{3}{|c|}{2005} & \multicolumn{3}{|c|}{2008} \\
\hline & $\begin{array}{r}\text { total } \\
(\%)\end{array}$ & $\begin{array}{r}\text { client } \\
\text { type }\end{array}$ & $\begin{array}{l}\text { peer } \\
\text { type }\end{array}$ & $\begin{array}{r}\text { total } \\
(\%)\end{array}$ & $\begin{array}{r}\text { client } \\
\text { type }\end{array}$ & $\begin{array}{l}\text { peer } \\
\text { type }\end{array}$ \\
\hline TCP & 97.43 & 94.93 & 97.66 & 96.00 & 95.51 & 96.06 \\
\hline$(<1024)$ & 13.99 & 58.93 & 8.66 & 17.98 & 76.16 & 11.35 \\
\hline 80 (http) & 9.32 & 50.78 & 5.54 & 14.06 & 64.96 & 8.26 \\
\hline 554 (rtsp) & 0.38 & 2.44 & 0.19 & 1.36 & 8.21 & 0.58 \\
\hline 443 (https) & 0.30 & 1.45 & 0.19 & 0.58 & 1.63 & 0.46 \\
\hline 20 (ftp-data) & 0.93 & 1.25 & 0.90 & 0.24 & 0.17 & 0.25 \\
\hline $81(-)$ & 0.15 & 0.04 & 0.16 & 0.23 & 0.04 & 0.25 \\
\hline $82(-)$ & 0.05 & 0.01 & 0.06 & 0.19 & 0.01 & 0.21 \\
\hline $110($ pop3) & 0.17 & 1.00 & 0.10 & 0.14 & 0.46 & 0.10 \\
\hline $22(\operatorname{ssh})$ & 0.09 & 0.17 & 0.10 & 0.10 & 0.16 & 0.09 \\
\hline 25 (smtp) & 0.14 & 0.51 & 0.11 & 0.07 & 0.11 & 0.07 \\
\hline $1000(-)$ & 0.03 & 0.02 & 0.04 & 0.07 & 0.01 & 0.08 \\
\hline others & 2.52 & 1.26 & 1.27 & 0.94 & 0.40 & 1.00 \\
\hline$(>=1024)$ & 83.44 & 36.00 & 89.00 & 78.02 & 19.35 & 84.71 \\
\hline 6346 (gnutella) & 0.92 & 0.84 & 0.93 & 0.94 & 0.67 & 0.97 \\
\hline 6699 (winmx) & 1.40 & 1.14 & 1.43 & 0.68 & 0.24 & 0.73 \\
\hline 7743 (winny) & 0.48 & 0.15 & 0.51 & 0.30 & 0.04 & 0.33 \\
\hline 1935 (rtmp) & 0.20 & 0.81 & 0.14 & 0.22 & 0.73 & 0.16 \\
\hline 6881 (bittorrent) & 0.25 & 0.06 & 0.27 & 0.22 & 0.02 & 0.24 \\
\hline $7144(-)$ & - & 0.02 & 0.03 & 0.19 & 0.03 & 0.21 \\
\hline $8080(-)$ & 0.11 & 0.15 & 0.14 & 0.11 & 0.09 & 0.15 \\
\hline 4662 (edonkey) & 0.12 & 0.02 & 0.13 & 0.13 & 0.00 & 0.14 \\
\hline $11560(-)$ & 0.03 & 0.02 & 0.10 & 0.04 & 0.01 & 0.11 \\
\hline $3074(-)$ & 0.02 & 0.03 & 0.10 & 0.02 & 0.32 & 0.07 \\
\hline others & 79.91 & 32.76 & 85.22 & 75.17 & 17.20 & 81.60 \\
\hline UDP $\quad *$ & 1.38 & 3.41 & 1.19 & 1.94 & 2.50 & 1.88 \\
\hline $53(\mathrm{dns})$ & 0.03 & 0.14 & 0.02 & 0.04 & 0.12 & 0.03 \\
\hline others & 1.35 & 3.27 & 1.17 & 1.90 & 2.38 & 1.85 \\
\hline ESP & 1.09 & 1.35 & 1.06 & 1.93 & 1.85 & 1.94 \\
\hline GRE & 0.07 & 0.12 & 0.06 & 0.09 & 0.08 & 0.09 \\
\hline ICMP & 0.01 & 0.05 & 0.01 & 0.02 & 0.05 & 0.02 \\
\hline others & 0.02 & 0.14 & 0.02 & 0.02 & 0.01 & 0.01 \\
\hline
\end{tabular}

When the total traffic in 2005 is compared with that in 2008, port 80 (http) accounted only for $9 \%$ in 2005 , and increased to $14 \%$ in 2008 . TCP dynamic ports accounted for $83 \%$ in 2005 and decreased to $78 \%$ in 2008 . That is, $5 \%$ shifted from dynamic ports to port 80 . Still, $78 \%$ of the total traffic in 2008 is between dynamic ports and most of them are considered to be peer-to-peer traffic. Hence, assuming the observed gradual shift from dynamic ports to well-known ports, user-to-user traffic will continue to be dominant in the coming years.

The usage of each dynamic port is tiny because recent peer-to-peer applications use arbitrary ports. The largest one, port 6699 in 2005 is only $1.4 \%$ and port 6346 in 2008 is only $0.9 \%$; it is no longer possible to make use of port numbers for identifying applications.

For the client-type users, port 80 accounted for $51 \%$ in 2005 and increased to $65 \%$ in 2008. Dynamic ports accounted for $36 \%$ in 2005 and decreased to $19 \%$ in
2008. Port 80 is much larger in this group, and $17 \%$ shifted from dynamic ports to port 80 and other wellknown ports. Also, there is a noticeable increase in port 554 used for the Real Time Streaming Protocol (RTSP) from $2 \%$ to $8 \%$. RTSP is a control protocol for streaming content employed by major streaming services. RTSP has the interleaved mode where the video data stream is interleaved on the original TCP control connection. Thus, the increase in port 554 is an evidence of increased video content. In fact, video streaming servers are often configured to support multiple transports and to switch protocols and ports according to clients' requests or when the port is blocked by firewalls. Thus, many of video contents are also transported over port 80 so that considerable video volume is supposed to be included in port 80. It is almost impossible to quantify the exact video volume in port 80 from Sampled NetFlow data but, given the heavy-tailed size distribution of video content which is much larger than traditional web content[10], it is likely that large part of the traffic on port 80 is already video and other rich media content.

Figure 10 compares temporal behaviors of three port groups: port 80, well-known ports other than port 80 , and dynamic ports for the total users, the client-type users and the peer-type users in 2005 and 2008. The volume is normalized to the peak value of the total traffic size not to reveal the absolute traffic volume of the ISP.

As expected, the total traffic is heavily affected by the peer-type user group. For the client-type users, it is clear that port 80 has increased and dynamic ports have decreased. Port 554 has a temporal pattern similar to port 80 but not shown in the plots.

For the peer-type users, most traffic is in dynamic ports, and a slight increase in port 80 is also observed. The dynamic port traffic gradually decreases after midnight towards morning with the bottom at around 8am, in contrast to the port 80 traffic that drops quickly after midnight with the bottom at around 4am. The slow decrease of the dynamic port traffic is probably caused by automatic dissemination of manually uploaded files over the night in file-sharing systems. Another plausible explanation is that file-sharing users stop the application when they finish the download or wake up in the morning instead of leaving it running all the time. This limited usage pattern of peer-to-peer file-sharing is partly due to an increasing number of ISPs imposing limits on bandwidth usage, and partly due to users' risk awareness about file-sharing applications as many information leakage incidents caused by compromised file-sharing applications have been reported in Japan.

For ordinary users, peer-to-peer file-sharing becomes less attractive with the advent of rich content services and their easy-to-use applications. At the same time, peer-to-peer traffic is still dominant in volume and will 

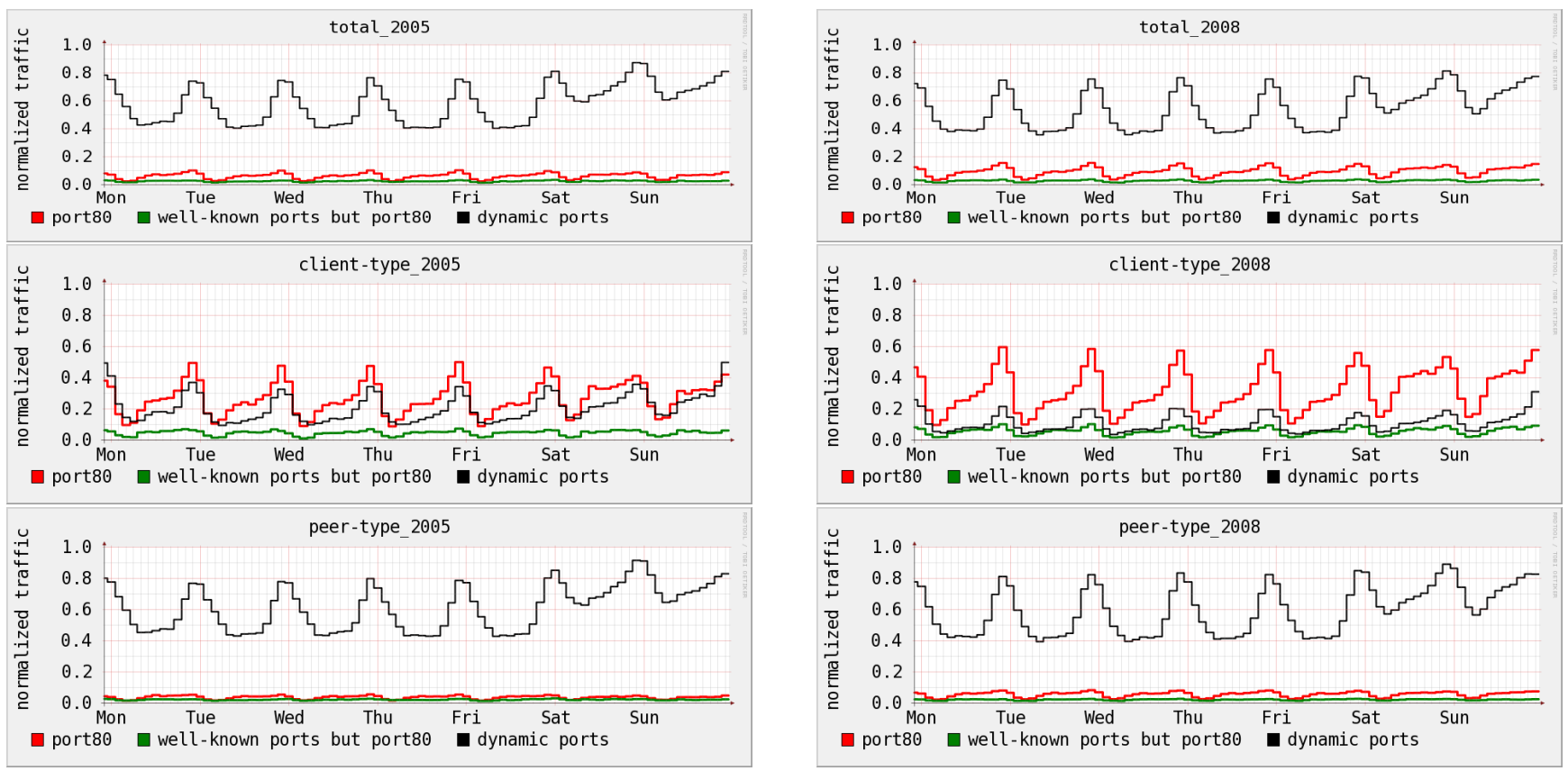

Figure 10: Temporal behavior of port usage: total users (top), client-type users (middle) and peertype users (bottom) in 2005 (left) and 2008 (right)

not go away anytime soon. Also, the peer-to-peer mechanism itself could evolve into a powerful engine to drive content distribution in large scale, if it becomes more friendly to users and ISPs[30].

\subsection{Summary}

The overall traffic is still dominated by heavy-hitters, mainly using peer-to-peer applications. However, their traffic decreased slightly in both population share and volume share.

The current slow growth rate is attributed to the fact that the dominant aggressive peer-to-peer traffic is not growing much. On the other hand, the client-type traffic is slowly moving towards high volume usage. The circumstantial evidence indicates that it is driven by video and other rich media content. The increase of video content is, however, not yet very visible in the total residential traffic volume at the macro level. We will examine the growth of this client-type user traffic in the next section.

Meanwhile, the capacity of the Internet, both access networks and core networks, will continue to grow. The annual growth of the access capacity is reported and projected to be $50 \%$ per year by the FTTH Council Europe[7] and, in fact, Japanese residential users are shifting from DSL to fiber access. The backbone networks also have been increasing capacity fast enough to handle the demands. TeleGeography[28] reports that annual international Internet capacity growth was $45 \%$ in 2004-2006 and $68 \%$ in 2007. We have also heard from the participating Japanese ISPs that they now have more headroom in the backbone capacity than 5 years ago when peer-to-peer applications were catching popularity.

If these trends continue, the traffic growth will stay at a modest rate comparable to the capacity growth. Video traffic is steadily growing but unlikely to disastrously overflow the global Internet in the short term.

\section{DISCUSSION}

Video and rich media content in residential traffic will have a significant impact on the traffic growth in the future[5]. Hence, we focus on the client-type users and analyze their outbound traffic as this group is characterized by download volume.

From the PDFs in Figure 6, traffic volume per user for the client-type group is roughly lognormally distributed. Because the distribution has a long tail, its simple arithmetic mean is heavily affected by the tail of the distribution. The mean is of interest as it is directly related to the total traffic volume. We examine the traffic characteristics using the properties of lognormal distributions.

It is not surprising that per-customer traffic volume follows a lognormal distribution as a multiplicative stochastic process leads to a lognormal distribution[9], and traffic growth is well modeled by a multiplicative stochastic process; each user increases traffic following a 
growth rate which is an independent identically distributed random process. Similar observations were reported in the distribution of traffic volume at an organizational level[8] as well as in a growth model of web pages[13].

The probability density function of a log-normal distribution is given by

$$
p(x)=\frac{1}{x \sigma \sqrt{2 \pi}} \exp \left(\frac{-(\ln x-\mu)^{2}}{2 \sigma^{2}}\right)
$$

where $\mu$ and $\sigma$ are the mean and standard deviation of $\ln (x)$. The mean of the original distribution, $p(x)$, is

$$
E(x)=\exp \left(\mu+\sigma^{2} / 2\right)
$$

and the variance of $p(x)$ is $\exp \left(2 \mu+\sigma^{2}\right)\left(\exp \left(\sigma^{2}\right)-1\right)$.

By fitting a lognormal distribution to the outbound client-type traffic from 2004 to 2008 with the nonlinear least-square Marquardt-Levenberg algorithm, we estimated $\mu$ and $\sigma$, and then, computed the mode $\exp (\mu)$ and the mean $E(x)$ in Table 5 using Equation 2.

Table 5: Growth of outbound traffic per clienttype user (MB/day)

\begin{tabular}{l|rr}
\hline & mode & mean \\
& $\exp (\mu)$ & $E(x)$ \\
\hline 2004 Apr & 26.2 & 110.6 \\
2005 Feb & 32.0 & 162.3 \\
2007 Jul & 65.7 & 483.2 \\
2008 Jun & 94.1 & 862.7 \\
\hline growth/yr & 1.38 & 1.72 \\
\hline
\end{tabular}

The mode of $94 \mathrm{MB} /$ day in 2008 seems reasonable considering popular web sites with rich media content (e.g., the mean file size of YouTube videos is $10 \mathrm{MB}[10]$, and popular applications such as Google Maps proactively fetch images in background). However, the mean of $863 \mathrm{MB} /$ day in 2008 seems much larger than one would expect; it is due to the influence from the tail of the distribution.

The annual growth rate of the mode is 1.38 and that of the mean is 1.72 obtained by fitting an exponential model by the least-square method. The growth rate of the mean is much larger than that of the mode as characterized by Equation 2 where the mean grows much faster than the mode. Also, $\sigma$ slightly increased from 2004 to 2008, which means larger contributions from the tail of the distribution.

Simplistic growth projections using both additive and multiplicative growth are shown in Table 6. Although traffic growth has been proved to be multiplicative, additive growth is shown as the lower bounds. With multiplicative growth, the mode and the mean in 2011 will be $248 \mathrm{MB} /$ day and $4.4 \mathrm{~GB} /$ day.

These growth rates are probably overestimated as 2004 and 2005 are before popular video services took off. Since the distribution is long-tailed, the mean is
Table 6: Simplistic growth projections for outbound traffic per client-type user (MB/day)

\begin{tabular}{l|rr|cr}
\hline & \multicolumn{2}{|c|}{ additive growth } & \multicolumn{2}{c}{ multiplicative growth } \\
& mode & mean & mode & mean \\
& $\exp (\mu)$ & $E(x)$ & $\exp (\mu)$ & $E(x)$ \\
\hline 2009 Jun & 110 & 1033 & 130 & 1480 \\
2010 Jun & 126 & 1203 & 179 & 2540 \\
2011 Jun & 142 & 1373 & 248 & 4359 \\
\hline
\end{tabular}

dictated by a small segment of users at the tail and becomes less predictable than the mode. We also expect a slowdown in the growth rate of the mean due to a larger bias against heavy-hitters as traffic volume approaches to the access link capacity. In addition, bandwidth caps introduced by ISPs affect the behavior of users. More and more Japanese ISPs have bandwidth cap policies for low-priced residential access services, explicitly by upload volume (e.g., 30GB/day by OCN and $15 \mathrm{~GB} /$ day by IIJ) or, in some cases, implicitly by p2p traffic shaping. When such policies were announced, many heavy-hitters reduced their usage although some uncompromising users simply migrated to other ISPs which do not have bandwidth caps.

There is a potential that traffic of ordinary users could grow to a substantial volume. The growth looks significant but it is similar to how the web traffic growth looked in the late 1990s when people were still using dial-up access. From an optimistic view, technical advances in access and core networks are likely to offset the traffic growth of this level.

\section{CONCLUSION}

As the Internet becomes a social communication platform, individual users of all ages start actively participating in digital communications with diverse content, notably with much richer images and videos than traditional web content. The traffic mix and volume are changing accordingly.

It is difficult to predict future traffic. As we have witnessed in the past, the advent of new applications would change traffic mix and, accordingly, growth rate. However, at the macro level, traffic will keep growing.

There will be many challenges posed by unprecedented traffic volume. A variety of technical solutions exist to mitigate possible problems such as content caching, content distribution networks and preferential quality of service. It also involves with economic factors such as the access service cost and the costs of backbone capacity and equipment as well as political and social factors such as net-neutrality and content management. Technical and solid observations on traffic are essential to understand changes and to adapt to new demands.

In this paper, we have shown that the apparent slow traffic growth is due primarily to the stalled growth of 
peer-to-peer traffic that is still dominant in the current traffic. At the same time, the usage of ordinary users is slowly swelling with increased diversity, driven by video and other rich media content. At the macro level, video traffic is still much smaller than peer-to-peer traffic, and will not significantly raise the traffic growth rate in the next few years.

As for the generality of our results, some aspects are specific to Japanese traffic such as the high penetration of fiber access, geographic concentration and language barriers. Nevertheless, our key findings seem to be common to other countries, although the exact ratio of traffic mix and their growth should be different from country to country, and probably, from ISP to ISP.

Persistent and consistent data collection is essential for this type of study. We will continue monitoring traffic by collecting aggregated traffic logs from the participating ISPs, and by analyzing per-customer traffic, hopefully from more ISPs.

\section{Acknowledgments}

We are grateful to the following ISPs for their support and assistance with our data collection; IIJ, SoftBank Telecom, K-Opticom, KDDI, NTT Communications, and SoftBank BB. We would like to thank the Ministry of Internal Affairs and Communications of Japan for their support in coordinating our study. We would also like to thank Jun Murai of the WIDE project, Andrew Odlyzko of University of Minnesota, and the anonymous reviewers for their valuable input. K. Fukuda is partially supported by the Grant-in-Aid for Young Scientists (B) from MEXT Japan.

\section{REFERENCES}

[1] N. Basher, A. Mahanti, A. Mahanti, C. Willamson, and M. Arlitt. A comparative analysis of web and peer-to-peer traffic. In $W W W^{\prime} 08$, pages $287-296$, Beijing, China, Apr. 2008.

[2] N. Brownlee and kc claffy. Understanding internet traffic streams: Dragonflies and tortoises. IEEE Communications Magazine, 40:110-117, 2002.

[3] K. Cho, K. Fukuda, H. Esaki, and A. Kato. The impact and implications of the growth in residential user-to-user traffic. In SIGCOMM2006, pages 207-218, Pisa, Italy, Aug. 2006.

[4] Cisco Sampled NetFlow. http://www.cisco.com/go/netflow.

[5] Cisco. Approaching the Zettabyte Era, white paper, June 2008.

[6] Cisco. Cisco Visual Networking Index - Forecast and Methodology, 2007-2012, white paper, June 2008.

[7] FTTH Council Europe. True broadband in Europe, Mar. 2008.

[8] K. Fukuda. Towards modeling of traffic demand of node in large-scale network. In IEEE ICC2008, pages 214-218, Beijing, China, May 2008.

[9] R. Gibrat. Les inégalitś économiques. Paris, Sirey, 1931.

[10] P. Gill, M. Arlitt, Z. Li, and A. Mahanti. Youtube traffic characterization: A view from the edge. In IMC2007, pages 15-28, San Diego, CA, Oct. 2007.

[11] K. P. Gummadi, R. J. Dunn, S. Saroiu, S. D. Gribble, H. M. Levy, and J. Zahorjan. Measurement, modeling, and analysis of a peer-to-peer file-sharing workload. In SOSP-19, pages 314-329, Bolton Landing, NY, Oct. 2003.

[12] L. Guo, S. Chen, Z. Xiao, E. Tan, X. Ding, and $\mathrm{X}$. Zhang. Measurements, analysis, and modeling of bittorrent-like systems. In IMC2005, pages 35-48, Berkeley, CA, Oct. 2005.

[13] B. A. Huberman and L. A. Adamic. Growth dynamics of the world-wide web. Nature, 401:131, 1999.

[14] W. John, S. Tafvelin, and T. Olovsson. Trends and differences in connection-behavior within classes of internet backbone traffic. In PAM2008 (LNCS 4979), pages 192-201, Cleveland, OH, Apr. 2008.

[15] Japan Internet Exchange Co., Ltd. http://www.jpix.co.jp/.

[16] Multifeed JPNAP service. http://www.jpnap.net/.

[17] M. Li, M. Claypool, R. Kinicki, and J. Nichols. Characteristics of streaming media stored on the web. ACM Transactions on Internet Technologies, 5(4):601-626, Nov. 2005.

[18] NSPIXP. http://nspixp.wide.ad.jp/.

[19] A. M. Odlyzko. Minnesota Internet traffic studies. http://www.dtc.umn.edu/mints/home.html.

[20] OECD Broadband Statistics. http://oecd.org/sti/ict/broadband, Dec. 2007.

[21] T. Oetiker. RRDtool: Round Robin Database Tool. http://ee-staff.ethz.ch/ oetiker/webtools/rrdtool/.

[22] T. Oetiker. MRTG: The multi router traffic grapher. In USENIX LISA, pages 141-147, Boston, MA, Dec. 1998.

[23] L. Plissonneau, J.-L. Costeux, and P. Brown. Analysis of peer-to-peer traffic on ADSL. In PAM2005 (LNCS3431), pages 69-82, Boston, MA, Mar. 2005.

[24] S. Saroiu, P. K. Gummadi, and S. D. Gribble. A measurement study of peer-to-peer file sharing systems. In $M M C N^{\prime} 02$, pages 156-170, San Jose, CA, Jan. 2002.

[25] S. Sarvotham, R. Riedi, and R. Baraniuk. Connection-level analysis and modeling of network traffic. In $I M W^{\prime} 01$, pages 99-103, San Francisco, CA, Nov. 2001.

[26] S. Sen and J. Wang. Analyzing peer-to-peer traffic across large networks. In IMW'02, pages 137-150, Marseille, France, Nov. 2002.

[27] M. Siekkinen, D. Collange, G. Urvoy-Keller, and E. W. Biersack. Performance limitations of adsl users: A case study. In PAM2007 (LNCS 4427), pages 145-154, Louvain-la-neuve, Belgium, Apr. 2007.

[28] TeleGeography Research. Globel Internet Geography. , 2007.

[29] The Ministry of Internal Affairs and Communications of Japan. MIC Statistics on data communications. http:

//www.soumu.go.jp/joho_tsusin/eng/statistics.html.

[30] H. Xie, Y. R. Yang, A. Krishnamurthy, Y. Liu, and A. Silberschatz. P4P: Provider portal for applications. In SIGCOMM 2008, Seattle, WA, Aug. 2008.

[31] YouTube, LLC. http://www.youtube.com/.

[32] Y. Zhang, L. Breslau, V. Paxson, and S. Shenker. On the characteristics and origins of internet flow rates. In SIGCOMM 2002, pages 309-322, Pittsburgh, PA, Aug. 2002. 